AUTORES:

1. Poloni RL, Tavares MCGF, Ferreira EL (2005). As funções da experiência corporal da dança em cadeira de rodas: perspectivas dos professores e alunos. In: Anais do IV Simpósio Internacional de Dança em cadeiras de rodas 50-59.

2. Antunes M. R (2006). Corpos que dançam com as diferenças. Mostra Virtual do 70 Salão de Extensão. UFGRS. Rio Grande do Sul.

3. Tolocka, R (2006). Aprendizagem e Dança com grupos heterogêneos. In: Dança e Diversidade Humana/ Rute Tolocka, Rosangela Velengia (org). São Paulo: Papirus.

4. Michaelis (1998). Moderno dicionário da língua portuguesa. São Paulo: Companhia Melhoramentos, (Dicionários Michaelis). 2259p.

5. Nanni D (2003). Dança - Educação - pré-escola à universidade - Rio de Janeiro: 4ed. Sprint.

6. Fonseca V (1995). Introdução às dificuldades de aprendizagem. Porto Alegre: Artes Médicas.

7. Gallahue D, Ozmun D (2003). Compreendendo o desenvolvimento motor: bebês, crianças, adolescentes e adultos. São Paulo: Phorte

8. Gariba CMS (2005). Dança Escolar: uma linguagem possível na educação física. Revista Digital, Bueno Aires, 10 (85)

9. Nascimento FM, Santos JC, Santos EV (2007). Brincando de dançar: contribuiçõos da dança na $2^{\mathrm{a}}$ infância. Pelotas: ESEF/UF Pelotas (Trabalho de Conclusão de Curso). 10. Zambom LF, Oliveira MS, Wagner MF (2006). A técnica da economia de fichas no transtorno de déficit de atenção e hiperatividade. Revista digital - 0 portal dos psicólogos 1-9.

11. Batistella PA, Krug MR, Oliveira L, Zancan RF (2002). Dança e desenvolvimento de portadores de necessidades educativas especiais- PNEEs. Revista Cadernos de ducação Especial, Santa Maria, (20): 89-95.

12. Freitas NK, Pereira JA (2007). Necessidades Educativas Especiais, arte, educação e inclusão. Revista E-Curriculum, São Paulo, 2(20): 1-18.

13. Santos RC, Figueiredo VMC (2003). Dança e inclusão no contexto escolar, um diálogo possível. Pensar a prática, (6): 107-116.

RESUMO
Giselle da Silva Tavares ${ }^{1}$

Aptidão física relacionada

Monalisa da Silva Reis

André de Araújo Pinto

Suzy Silva Pinto ${ }^{1}$

Romina K. S. Michiles ${ }^{1}$

Lionela da Silva Corrêa

Universidade Federal do Amazonas, Brasil

ao desempenho motor

e estado nutricional de

adolescentes de 11 e 12 anos

de uma escola pública

\section{de Manaus}

PALAVRAS CHAVE:

Estado nutricional. Desempenho motor.

Escolares.

A adolescência é uma fase de transição da infância para a fase adulta sendo marcada por grandes modificações biopsicossociais; neste período vulnerável, os hábitos alimentares e os padrões de inatividade física podem contribuir para o aumento da incidência de obesidade e dos distúrbios nutricionais. 0 objetivo do estudo foi avaliar o desempenho motor e estado nutricional de adolescentes de 11 e 12 anos de uma escola pública de Manaus. A amostra foi composta por 38 adolescentes, dos quais 20 eram do sexo feminino e 18 do sexo masculino. 0 instrumento metodológico utilizado foi a bateria de teste do PROESP-BR contendo 6 itens: teste de velocidade (corrida de velocidade); teste de agilidade (quadrado); teste de potência de membros inferiores (salto horizontal); teste de potência de membros superiores (arremesso de medicineball); resistência cardiorrespiratória (corrida de 6 minutos) e peso/altura ${ }^{2}$ (IMC). Os resultados apontaram para um nível abaixo do nível desejado de aptidão física, apresentando assim um nível fraco. Conclui-se que grande parte dos adolescentes apresentou um déficit no nível de atividade física retratado pela aptidão fraca. 
Relationship of physical fitness with

\section{motor performance and nutritional status}

in adolescents aged between 11 and 12 years

\section{old at a public school in Manaus}

\section{ABSTRACT}

Adolescence is a stage of transition from childhood to adulthood and is marked by large biopsychosocial changes. In this vulnerable period, poor eating habits and patterns of physical inactivity may contribute to an increased incidence of obesity and nutritional disorders. The aim of the study was to evaluate the motor performance and nutritional status of adolescents aged between 11 and 12 years old, at a public school in Manaus. The sample consisted of 38 adolescents, of whom 20 were female and 18 were male. The methodological tool used was the battery PROESP - BR, containing 6 items: Speed Test (sprint), agility test (square), lower limb power (standing long jump) Test, Power Test of upper limbs (pitch medicine ball), cardiorespiratory endurance (running 6 minutes) and weight/height2 (BMI) . The results pointed to a level below the desired level of fitness as well with a low level. It is concluded that most adolescents showed a deficit at the level of physical activity portrayed by poor fitness.

\section{KEY WORDS}

Nutritional status. Motor performance. Students.

\section{INTRODUÇÃo}

A adolescência é uma fase de transição da infância para a fase adulta sendo marcada por grandes modificações biopsicossociais ${ }^{1}$; neste período vulnerável, os hábitos alimentares e os padrões de inatividade física podem contribuir para o aumento da incidência de obesidade e dos distúrbios nutricionais ${ }^{2}$.

Verifica-se um aumento significativo do excesso de peso/obesidade na infância e na adolescência. Os índices de obesidade triplicaram num período de 6 anos para a faixa etária entre 6 e 19 anos 3,4

Crianças e adolescentes identificados com algum tipo de dificuldade motora também tendem a apresentar um IMC elevado ${ }^{3}$.

A prática de atividades físicas regulares auxilia no bom desempenho das funções vitais das pessoas em todas as idades. Os baixos níveis de atividade física em adolescentes estão associados a fatores, como: demográficos, socioeconômicos e estilo de vida (hábitos alimentares) ${ }^{5}$.

Durante o processo de crescimento e desenvolvimento biológico do ser humano, ocorre uma série de mudanças em sua constituição física, representadas pelas modificações nas características antropométricas das dimensões, estrutura e composição corporais ${ }^{6}$.

Em crianças e adolescentes, um nível elevado de atividade física contribui para melhorar o perfil lipídico e metabólico, reduzindo a prevalência de obesidade. Além disso, é provável que uma criança fisicamente ativa se torne um adulto ativo. Promover a atividade física na infância e na adolescência significa estabelecer uma base solida para a redução da prevalência do sedentarismo na idade adulta ${ }^{7}$.

Desta forma, este estudo teve como objetivo avaliar o desempenho motor e estado nutricional de adolescentes de 11 e 12 anos de uma escola pública de Manaus.

\section{MATERIAIS E MÉTODOS}

Esse estudo caracteriza-se como pesquisa descritiva com abordagem quantitativa sendo aquela que o pesquisador apenas registra e descreve os fatos sem intervir ${ }^{8}$.

\section{AMOSTRA}

A amostra foi composta por 38 adolescentes sendo 20 do gênero feminino e 18 do gênero masculino, para a seleção foi utilizada a estatística não probabilística por cotas. 


\section{INSTRUMENTO}

\section{E PROCEDIMENTOS}

0 instrumento utilizado para medir o estado nutricional e o desempenho motor dos adolescentes foi à bateria de teste e medidas PROESP-BR versão 2012, que foi desenvolvida para a avaliação de parâmetros de saúde e desempenho motor para uso independente de suas condições de trabalho, é uma bateria de baixo custo, que visa ajudar as escolas brasileiras com carências em suas estruturas e precária de matérias para as aulas.

0 instrumento é constituído com os seguintes testes, que estão relacionados ao desempenho motor: Força explosiva de membros superiores (arremesso de medicineball $2 \mathrm{~kg}$ ), Força explosiva de membros inferiores (salto horizontal), Agilidade (teste do quadrado), Velocidade (corrida de 20 metros) e Aptidão cardiorrespiratória (corrida de 6 minutos) e composição corpórea (IMC)

Portanto, foi necessário selecionar instrumentos de medidas e avaliação de muito baixo custo, com o mínimo de materiais sofisticados, de fácil acesso e aplicação, evidentemente cumprindo rigorosamente as exigências de validade, fidedignidade e objetividade 9 .

0 teste foi aplicado na seguinte ordem (conforme o manual PROESP):

1) MASSA CORPORAL TOTAL

Material: uma balança com precisão de até 500 gramas.

A medida foi anotada em quilogramas, com a utilização de uma casa decimal.

2) ESTATURA

Material: trena métrica com precisão de $2 \mathrm{~mm}$.

A medida da estatura foi anotada em centímetros, com uma casa decimal.

Medida do Índice de Massa Corporal (IMC)

Orientação: é determinado através do cálculo da razão entre a medida de massa corpo-

ral total em quilogramas pela estatura em metros elevada ao quadrado.

IMC $=$ massa $(\mathrm{kg}) /$ estatura $(\mathrm{m}) 2$

A medida foi anotada com uma casa decimal.

3) TESTE DE FORÇA EXPLOSIVA DE MEMBROS INFERIORES

Material: uma trena e uma linha traçada no solo.

A distância do salto foi registrada em centímetros, com uma decimal, a partir da linha traçada no solo até o calcanhar mais próximo desta.

4) TESTE DE FORÇA EXPLOSIVA DE MEMBROS SUPERIORES

Material: uma trena e um medicineball de $2 \mathrm{~kg}$

A medida foi registrada em centímetros, com uma casa decimal.

5) TESTE DE AGILIDADE

Material: um cronômetro, um quadrado desenhado em solo antiderrapante com $4 \mathrm{~m}$ de lado e quatro cones de $50 \mathrm{~cm}$ de altura.

A medida foi registrada em segundos e centésimos de segundos (duas casas após a vírgula)
6) TESTE DE VELOCIDADE

Material: um cronômetro e uma pista de 20 metros demarcada com três linhas paralelas no solo, da seguinte forma: a primeira (linha de partida); a segunda, distante $20 \mathrm{~m}$ da primeira (linha de cronometragem); e a terceira, marcada a um metro da segunda (linha de chegada). A terceira linha serve como referência de chegada para o aluno, na tentativa de evitar que ele inicie a desaceleração antes de cruzar a linha de cronometragem. Dois cones para a sinalização da primeira e terceira linhas.

O cronometrista registrou o tempo do percurso em segundos e centésimos de segundos (duas casas após a vírgula).

7) TESTE DE RESISTÊNCIA CARDIOVASCULAR

Material: local plano, com marcação do perímetro da pista.

Cronômetro e ficha de registro.

Os resultados foram anotados em metros, com aproximação em dezenas.

\section{METODOLOGIA}

O estudo obedeceu à metodologia do instrumento (PROESP-BR), tendo como base valores numéricos que definem o nível de desempenho motor e estado nutricional dos adolescentes conforme a idade. A análise dos dados deu-se por meio da estatística descritiva.

Em relação ao desempenho motor os itens são classificados em uma escala de graus de aptidão: fraco - razoável - bom - muito bom - excelente, e o estado nutricional foi classificado de acordo com o resultado do IMC (baixo peso, Sobrepeso e Obesidade).

\section{RESULTADOS}

Participaram do estudo 38 adolescentes, sendo 18 do sexo masculino e 20 do sexo feminino com idade entre 11 e 12 anos, conforme quadro 1 .

QUADRO 1 - Distribuição dos sujeitos por faixa etária

\begin{tabular}{|c|c|c|c|c|}
\hline \multirow[t]{2}{*}{ IDADE } & \multicolumn{2}{|c|}{ FREQUÊNCIA } & \multirow[t]{2}{*}{ TOTAL POR IDADE } & \multirow[t]{2}{*}{ PERCENTUAL } \\
\hline & MENINAS & MENINOS & & \\
\hline 11 ANOS & 11 & 4 & 15 & $39,47 \%$ \\
\hline 12 ANOS & 9 & 14 & 23 & $60,53 \%$ \\
\hline TOTAL & 20 & 18 & 38 & $100 \%$ \\
\hline
\end{tabular}

$145-\operatorname{RPCD} 14$ (S1.A) 
A partir dos resultados obtidos por meio da bateria de testes e medidas PROESP-BR foi possível avaliar o estado nutricional e o desempenho motor dos adolescentes. Os resultados dos componentes (IMC, potência de membros superiores, inferiores, agilidade, velocidade e aptidão cardiorrespiratória) foram analisados por meio da frequência e percentual.

0 estado nutricional assume grande importância na determinação da saúde de sujeitos. A desnutrição, o sobrepeso e a obesidade são estados nutricionais referentes a problemas que podem afetar o adequado desenvolvimento e interferir nos processos de saúde/doença ${ }^{10}$.

No índice de massa corporal - IMC 60,53\% dos adolescentes enquadraram-se como eutróficos ou peso normal, $34,21 \%$ apresentaram sobrepeso e $5,26 \%$ baixo peso como mostra o quadro 2 .

QUADRO 2 - Resultado do teste de IMC

\begin{tabular}{|c|c|c|c|c|}
\hline $\begin{array}{c}\text { COMPONENTES } \\
\text { DA APTIDÃO FÍSICA IMC }\end{array}$ & $\begin{array}{l}\text { MENINAS } \\
\text { N (\%) }\end{array}$ & $\begin{array}{l}\text { MENINOS } \\
\text { N (\%) }\end{array}$ & $\begin{array}{c}\text { TOTAL } \\
N\end{array}$ & $\%$ \\
\hline BAIXO PESO & $1(5,0 \%)$ & $1(5,5 \%)$ & 2 & $5,26 \%$ \\
\hline SOBREPESO & $7(35,0 \%)$ & $6(33,3 \%)$ & 13 & $34,21 \%$ \\
\hline EUTRÓFICOS & $12(60,0 \%)$ & $11(61,1 \%)$ & 23 & $60,53 \%$ \\
\hline
\end{tabular}

Em relação à potência muscular de membros inferiores, componente de aptidão relacionada ao desempenho, 42, 11 \% dos adolescentes apresentaram um nível muito bom, 15,79\% dos adolescentes apresentaram nível bom, 7,89\% dos adolescentes apresentaram nível excelente, 13,16\% dos adolescentes apresentaram nível razoável e 21,05 \% apresentaram nível fraco como mostra o quadro 3.

Na potência muscular de membros superiores, observou-se que $86,84 \%$ dos adolescentes apresentaram nível fraco, no entanto 7,89\% apresentaram nível razoável e 5,26\% apresentaram nível bom.

Com relação à aptidão cardiorrespiratória, 39,47\% dos adolescentes apresentaram nível fraco de aptidão, 10,53\% apresentaram nível bom e razoável na mesma proporção, 15,79\% apresentaram nível muito bom e 23,68\% apresentaram nível excelente.

No teste de agilidade, $47,37 \%$ dos adolescentes apresentaram nível fraco, 13,16\% apresentaram nível bom e muito bom na mesma proporção, 15,79\% observou-se desempenho excelente e 10,53\% apresentaram nível razoável.

Em relação ao teste de velocidade, $39,47 \%$ dos adolescentes apresentaram nível fraco 2,63\% apresentaram nível bom e excelente na mesma proporção 31,58\% apresentaram nível muito bom e 23,68 apresentaram nível razoável.
QUADRO 3 - Resultado do teste de aptidão física relacionada ao desempenho.

\begin{tabular}{|c|c|c|}
\hline $\begin{array}{l}\text { COMPONENTES DA APTIDÃO FÍSICA } \\
\text { RELACIONADA AO DESEMPENHO }\end{array}$ & FREQUÊNCIA & PORCENTAGEM \\
\hline \multicolumn{3}{|c|}{ POTENCIA DE MEMBROS INFERIORES } \\
\hline FRACO & 8 & $21,05 \%$ \\
\hline BOM & 6 & $15,79 \%$ \\
\hline MUITO BOM & 16 & $42,11 \%$ \\
\hline EXCELENTE & 3 & $7,89 \%$ \\
\hline RAZOÁVEL & 5 & $13,16 \%$ \\
\hline \multicolumn{3}{|c|}{ POTENCIA DE MEMBROS SUPERIORES } \\
\hline FRACO & 33 & $86,84 \%$ \\
\hline BOM & 2 & $5,26 \%$ \\
\hline MUITO BOM & 0 & $0,00 \%$ \\
\hline EXCELENTE & 0 & $0,00 \%$ \\
\hline RAZOÁVEL & 3 & $7,89 \%$ \\
\hline \multicolumn{3}{|c|}{ APTIDÃO CARDIORRESPIRATÓRIA } \\
\hline FRACO & 15 & $39,47 \%$ \\
\hline BOM & 4 & $10,53 \%$ \\
\hline MUITO BOM & 6 & $15,79 \%$ \\
\hline EXCELENTE & 9 & $23,68 \%$ \\
\hline RAZOÁVEL & 4 & $10,53 \%$ \\
\hline \multicolumn{3}{|c|}{ AGILIDADE } \\
\hline FRACO & 18 & $47,37 \%$ \\
\hline BOM & 5 & $13,16 \%$ \\
\hline MUITO BOM & 5 & $13,16 \%$ \\
\hline EXCELENTE & 6 & $15,79 \%$ \\
\hline RAZOÁVEL & 4 & $10,53 \%$ \\
\hline
\end{tabular}




\begin{tabular}{ccc}
\hline $\begin{array}{c}\text { COMPONENTES DA APTIDÃO FÍSICA } \\
\text { RELACIONADA AO DESEMPENHO }\end{array}$ & FREQUÊNCIA & PORCENTAGEM \\
\hline \multicolumn{3}{c}{ VELOCIDADE } \\
\hline FRACO & 15 & $39,47 \%$ \\
\hline BOM & 1 & $2,63 \%$ \\
\hline MUITO BOM & 12 & $31,58 \%$ \\
\hline EXCELENTE & 1 & $2,63 \%$ \\
\hline RAZOÁVEL & 9 & $23,68 \%$ \\
\hline
\end{tabular}

Nos indicadores de desempenho motor a maior parte dos adolescentes não atingiram aos critérios da bateria de teste e medidas proesp-br nos seguintes testes: potência de membros superiores, aptidão cardiorrespiratória, agilidade e velocidade.

Percebe-se que um quantitativo dos adolescentes apresentaram desempenho fraco, tais resultados podem ser explicado pelo fato dos meninos sofrerem aumento relativo dos membros superiores por volta dos 13 anos, correspondendo ao aparecimento da puberdade, enquanto que as meninas demonstram níveis inferiores aos meninos, levando-se em conta a fraqueza de braços e ombros e/ou estilo de vida crescentemente sedentário ${ }^{22}$.

Um menor envolvimento com a prática de atividade física entre os adolescentes vem decrescendo nos últimos anos devido a uma tendência ao sedentarismo ${ }^{23}$, como assistir TV, navegar na internet, jogar vídeo game entre outros ${ }^{24}$.

Escolares entre 11 a 14 anos juntamente com crescimento somático, e consequente aumento da massa muscular que está ligada ao consumo de oxigênio, há o aumento dos órgãos fazendo com que aconteça a melhora da aptidão cardiorrespiratória ${ }^{25}$. Os níveis de aptidão física de crianças e adolescentes são influenciados pelas transformações fisiológicas e anatômicas decorrentes pela quantidade de homônimos que geralmente aumenta com a chegada da puberdade e pela quantidade de atividade física habitual ${ }^{15}$. É nesse período de vida que os adolescentes estão em processo de maturação, nesse período da puberdade acontece aumento da massa magra e logo um aumento da força muscular ${ }^{26}$.

Baixa aptidão física esta associados a elevados índices de colesterol e triglicerídeos, pressão arterial e risco maior a obesidade.

A desnutrição na infância e adolescência constitui uma condição que pode acarretar em problemas para os mesmos, pois ocorre a falta de gordura necessária para manutenção adequada das funções fisiológicas e, se presente, representa risco a saúde ${ }^{10}$.

Hábitos alimentares inadequados e estilo de vida tipicamente sedentário tem sido considerado um importante fator de risco à saúde em adultos, bem como em crianças e adolescentes, de ambos os sexos, em diferentes faixas etárias ${ }^{14}$.

Estudos têm apontado para um risco elevado de as crianças e adolescentes com sobrepeso e obesidade tornarem-se adultos obeso ${ }^{15}$. A obesidade também se apresenta como fator de risco para o desenvolvimento de outras doenças, como a síndrome metabólica ${ }^{16} \mathrm{e}$ as doenças cardiovasculares ${ }^{17}$, o que torna de extrema importância à elaboração de estratégias para o combate dessa desordem nutricionais.

Jovens com hábitos de vida pouco saudável durante a infância e a adolescência, incluindo desde baixos níveis de atividade física habitual até alimentação de baixo valor e alto valor energético, parecem possuir maior predisposição para o aparecimento de diversas disfunções metabólicas em idades precoces ${ }^{14}$.

0 excesso de gordura corporal, além de ser considerado fator de risco para o surgimento de doenças cardiovasculares, hipertensão e diabetes ${ }^{18}$. Muitas pesquisas ${ }^{19,20,21}$ relatam que o excesso de peso pode limitar os movimentos e sobrecarregar os ossos, músculos, articulações e os sistemas respiratório e circulatório, acarretando em menor resistência orgânica.

Conclui-se que grande parte dos adolescentes apresentaram um déficit no nível de atividade física retratado pela aptidão fraca, no entanto a maioria dos adolescentes apresentaram adequado estado nutricional e baixa prevalência de sobrepeso e obesidade.

Esses resultados podem ser reflexos do estilo de vida da sociedade, no qual cada vez mais os jovens escolhem atividades de lazer mais sedentária.

Faz-se necessário adotar estratégias de intervenção na escola pesquisa, uma vez que os fatores de risco apresentam-se quantitativo elevado. Sendo o trabalho interdisciplinar essencial, ou seja, todos os educadores da escola tem a responsabilidade de mudar esse quadro tão preocupante. 
1. Mota DCL, Laus MF, Almeida SS, Costa TMB, Moreira $\quad$ 12. PRADO, J.M. A criança em idade escolar em IlRCM (2012). Imagem corporal e suas relações com a habela: crescimento e atividade motora. 2005. Disseratividade física e o estado nutricional em adolescente. tação (mestrado) Universidade estadual de Campinas, PSICO, Porto Alegre, PUCRS, 43(2): 237-242.

2. Santos GG, Sousa JB, Toscano MB, Morais MAE (2011). Hábitos alimentares e estado nutricional de adolescentes de um centro de juventude da cidade de Anápolis. Ensaios e Ciências: Ciências Biológicas, Agrárias e da Saúde, 15(1): 141-151.

3.Contreira AR, Capistrano R, Oliveira AVP, Beltrama TS (2012). Avaliação psicomotora e nutricional de escolares de Florianópolis/SC. Biomotriz 6(2): 61-76. 4. Contreira AR, Capistrano R, Oliveira AVP, Beltrame TS (2013). Indicadores de saúde em escolares: avaliação estado nutricional e desempenho motor. 14 (1):13-17.

5. Oliveira G, Silva DAS, Maggi RM, Petroski EL, Farias JM (2012). Fatores sociodemográficos de aptidão física associados a baixos níveis de atividade física em adolescentes de uma cidade do sul do Brasil. Rev Educ. Fis/UEM 23(4): 635-645.

6. Ferreira M, Bohme MTS (1998). Diferenças sexuais no desempenho motor de crianças: influência da adiposidade corporal. Rev Paul Educação Física, São Paulo, 12 (2): 181-192.

7. Lazzoli JK, Nobrega ACL, Carvalho T, Oliveira MAB, Teixeira JAC, Leitão MB, Leite N, Meyer F, Drummond FA, Pessoa MSV, Rezende L, Rose EH, Barbosa ST, Magni JRT, Nahes RM, Michels G, Matsudo V (1998). Atividade física e saúde na infância e adolescência. Rev Brasileira Medicina do Esporte. 4(4): 1-3.

8. Gonçalves HA (2005). Manual de Metodologia da Pesquisa Científica. São Paulo: Avercamp.

9. Gaya A (2012). Manual do Projeto Esporte Brasil.

10. Teixeira HM, Marba RF, Cardoso MNM, Barbosa LCA, Pinto RF (2010). Desempenho motor de escolares em diferentes estágios nutricionais: avaliação do processo das habilidades motoras fundamentais: salto, chute e arremesso. Fiep bulletin, 80: 1-7.

11. GAYA, Adroaldo. Manual do Projeto Esporte Brasil, 2012.
21. Nahas MV (2010). Atividade Fisica, Saúde e Qualidade de Vida: conceitos e sugestões para um estilo de

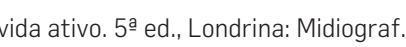

22. Gallahue DL, Ozmun JC (2005). Compreendendo o Desenvolvimento Motor: bebês crianças, adolescentes e adultos. São Paulo: Editora Phorte.

23. Guerra C, Reckziegel MB, Burgos MS (2008). Perfil somatomotor e indicadores de saúde de adolescentes com índices alterados de glicemia. Rev. Digital Cinergi, 9(1): 29-36.

24. Nobre FSS, Krebs RJ, Valentini NC (2009). Práticas de lazer, nível de atividade física e aptidão física de moças e rapazes Brasileiros. Rev salud pública, 11(5): 713-723.

25. Tomkinson GR, Omkinson GR, Olds TS (2007). Pediatric Fitness. Secular Trends and Geographic Variability. Med Sport Sci Basel, 50:67-90.

26. Bortoni WL, Bojikian LP (2007). Crescimento aptidão física de escolares do sexo masculino, participantes de programa de iniciação esportiva. Brazilian Journal of Biomotricity, 1(4):114-122. 